\title{
Statistical theory of light nucleus reactions with $1 p$-shell light nuclei
}

\author{
Sun Xiaojun ${ }^{1, a}$ and Zhang Jingshang ${ }^{2}$ \\ ${ }^{1}$ Guangxi Normal University, College of Physics, Guilin, Guangxi, China \\ 2 China Institute of Atomic Energy, Department of Nuclear Physics, Beijing, China
}

\begin{abstract}
The 1p-shell light elements ( $\mathrm{Li}, \mathrm{Be}, \mathrm{B}, \mathrm{C}, \mathrm{N}$, and $\mathrm{O}$ ) had long been selected as the most important materials for improving neutron economy in thermal and fast fission reactors and in the design of acceleratordriven spallation neutron sources. A statistical theory of light nucleus reactions (STLN) is proposed to describe the double-differential cross sections for both neutron and light charged particle induced nuclear reactions with 1p-shell light nuclei. The dynamics of STLN is described by the unified Hauser-Feshbach and exciton model, in which the angular momentum and parity conservations are strictly considered in equilibrium and pre-equilibrium processes. The Coulomb barriers of the incoming and outgoing charged particles, which significantly influence the open channels of the reaction, can be reasonably considered in incident channel and different outgoing channels. In kinematics, the recoiling effects in various emission processes are strictly taken into account. The analytical energy and angular spectra of the reaction products in sequential and simultaneous emission processes are obtained in terms of the new integral formula proposed in our recent paper. Taking ${ }^{12} \mathrm{C}(\mathrm{n}, \mathrm{xn}),{ }^{9} \mathrm{Be}(\mathrm{n}, \mathrm{xn}),{ }^{16} \mathrm{O}(\mathrm{n}, \mathrm{xn})$, and ${ }^{9} \mathrm{Be}(\mathrm{p}, \mathrm{xn})$ reactions as examples, we had calculated the double-differential cross sections of outgoing neutrons and compared with the experimental data. In addition, we had also calculated the partition and total kerma coefficients for ${ }^{12} \mathrm{C}(\mathrm{n}, \mathrm{xn})$ and ${ }^{16} \mathrm{O}(\mathrm{n}$, xn) reactions, respectively. The existing experimental data can be remarkably well reproduced by STLN, which had been used to set up file-6 in CENDL database.
\end{abstract}

\section{Introduction}

The 1p-shell light elements had long been selected as the most important materials for improving neutron economy in thermal and fast fission reactors and in the design of accelerator-driven spallation neutron sources. The doubledifferential cross sections (DDCS) of the 1p-shell light nuclei are the essential data for the designs of the nuclear facilities, such as International Fusion Materials Irradiation Facility (IFMIF), International Thermonuclear Experimental Reactor (ITER), Molten Salt Fast Reactor (MSFR), Accelerator Driven Advanced Nuclear Energy System (ADANES), and so on. File-6, one of the important files of the nuclear reaction database, is recommended when the energy and angular distributions of the emitted particles must be coupled, when it is important to give a concurrent description of neutron scattering and particle emission, when so many reaction channels are open that it is difficult to provide separate reactions, or when accurate distributions of the charged particle or residual nucleus are required for particle transport, heat deposition, or radiation damage calculations. [1] In a word, file- 6 can give the most detailed information of the emission particles.

However, there are some difficulties for the model calculations of DDCS with 1p-shell light nuclei. Firstly, there are particle emissions between the discrete levels of the residual nuclei, which have not been considered in many evaluation codes. Secondly, the recoiling effects of the residual nuclei must be considered because of their

a e-mail: sxj0212@gxnu.edu.cn light masses. Thirdly, every light nucleus has some specific features, and so on.

The structure of this paper is as follow. In Sect. 2, the dynamics and kinematics of Statistical Theory of Light Nucleus reaction are introduced. The calculated results are compared with the experimental data in Sect. 3. In Sect. 4, a summary is simply given.

\section{Statistical Theory of Light Nucleus reaction}

\subsection{Dynamics}

The dynamics of Statistical Theory of Light Nucleus reaction (STLN) is described by the unified HauserFeshbach and exciton model. The cross section of the first particle $m_{1}$ with kinetic energy $\varepsilon_{1}^{c}$ emitted from compound nucleus $M_{C}$ with excited energy $E^{*}$ to residual nucleus $M_{1}$ with $E_{k 1}$ energy level and recoil kinetic energy $E_{1}^{c}$ is expressed as [2]

$$
\begin{aligned}
\sigma_{m_{1}, k_{1}}\left(E_{n}\right)= & \sum_{j \pi} \sigma_{a}^{j \pi}\left(E_{n}\right) \sum_{n=3}^{n_{\max }}\left\{P^{j \pi}(n) \frac{W_{m_{1}, k}^{j \pi}\left(n, E^{*}, \varepsilon_{m_{1}}\right)}{W_{T}^{j \pi}\left(n, E^{*}\right)}\right. \\
& \left.+Q^{j \pi}(n) \frac{W_{m_{1}, k_{1}}^{j \pi}\left(E^{*}, \varepsilon_{m_{1}}\right)}{W_{T}^{j \pi}\left(E^{*}\right)}\right\}
\end{aligned}
$$

where $\sigma_{a}^{j \pi}$ is the absorption cross section, and the terms in the brace denote the contributions of the pre-equilibrium and equilibrium processes, respectively. And $P^{j \pi}(n)$ is the

(C) The Authors, published by EDP Sciences. This is an Open Access article distributed under the terms of the Creative Commons Attribution License 4.0 (http://creativecommons.org/licenses/by/4.0/). 


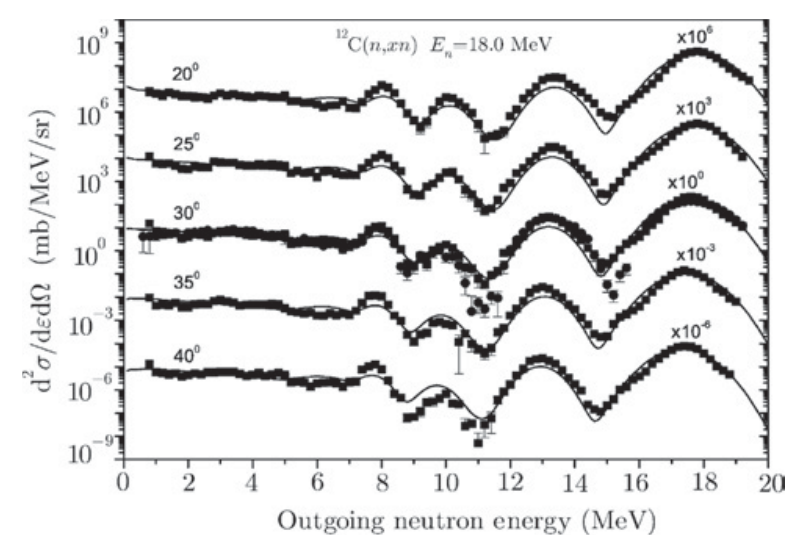

Figure 1. The double-differential cross sections for ${ }^{12} \mathrm{C}(\mathrm{n}, \mathrm{xn})$ at $E_{n}=18 \mathrm{MeV}$ for outgoing angles $20^{\circ}, 25^{\circ}, 30^{\circ}, 35^{\circ}, 40^{\circ}$, respectively [4].

occupation probability of the $n$-th exciton state in the $j \pi$ channel ( $j$ and $\pi$ denote the angular momentum and parity in the final state, respectively).

The cross section of the secondary particle $m_{2}$ with kinetic energy $\varepsilon_{2}^{c}$ emitted from $M_{1}$ with $E_{k 1}$ energy level to the residual nucleus $M_{2}$ with $E_{k 2}$ energy level and recoil kinetic energy $E_{2}^{c}$ is expressed as

$$
\sigma_{k_{1} \rightarrow k_{2}}\left(n, m_{1}, m_{2}\right)=\sigma_{k_{1}}\left(n, m_{1}\right) R_{m_{2}}^{k_{1} \rightarrow k_{2}}\left(E_{k_{1}}\right),
$$

where $R_{m_{2}}^{k_{1} \rightarrow k_{2}}\left(E_{k_{1}}\right)$ is the branching ratio of the secondary emitted particle $m_{2}$ from energy level $E_{k 1}$ of $M_{1}$ to the energy level $E_{k 2}$ of $M_{2}$.

\subsection{Kinematics}

The energy conservation for the first particle emission processes is expressed as

$$
\bar{\varepsilon}_{m_{1}}+\bar{E}_{M_{1}}+E_{k_{1}}=E_{n}+B_{0}-B_{1},
$$

where terms of the left side are the average energy of the first emitted particle and the first residual nucleus in the lab system, and the excited energy of the first residual nucleus, respectively. $E_{n}$ is the incident energy in lab system, and $B_{0}$ and $B_{1}$ are the binding energies of the incident particle and the first emitted particle in the compound nucleus, respectively.

For the secondary particle emission processes, the recoil nucleus system (RNS) is set on the first residual nucleus $M_{1}$, i.e., $M_{1}$ is in static state to emit secondary particle $m_{2}$ with definitive energy. In the frame of STLN, it is assumed that the double-differential cross section of the secondary emitted particle $m_{2}$ in RNS is the isotropic distribution as follows

$$
\frac{d^{2} \sigma}{d \varepsilon_{m_{2}}^{r} d \Omega_{m_{2}}^{r}}=\frac{1}{4 \pi} \delta\left[\varepsilon_{m_{2}}^{c}-\varepsilon_{m_{2}}^{r}\left(1+2 \gamma \cos \Theta+\gamma^{2}\right)\right] .
$$

Using a special integral formula [3], which has not been compiled in any integral tables or any mathematical softwares, i.e.,

$$
\begin{aligned}
& \int_{0}^{\pi} d t P_{l}\left(\sqrt{\left(1-\eta^{2}\right) \sin ^{2} \theta_{2}} \cos t+\eta \cos \theta_{2}\right) \\
& =\pi P_{l}(\eta) P_{l}\left(\cos \theta_{2}\right),
\end{aligned}
$$

so we can obtain the analytical energy and angular spectra, with $P_{l}$ the Legendre polynomial. And the energy

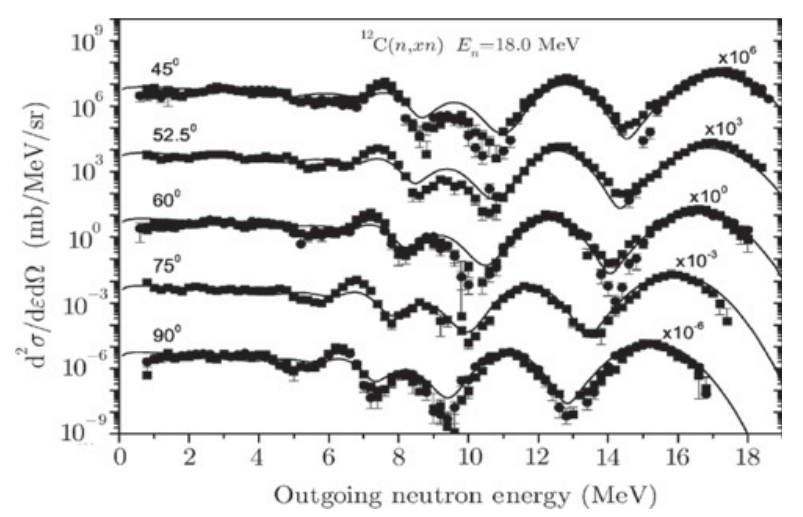

Figure 2. The same as Fig. 1, but for outgoing angles $45^{\circ}, 52.5^{\circ}$, $60^{\circ}, 75^{\circ}, 90^{\circ}$, respectively [4].

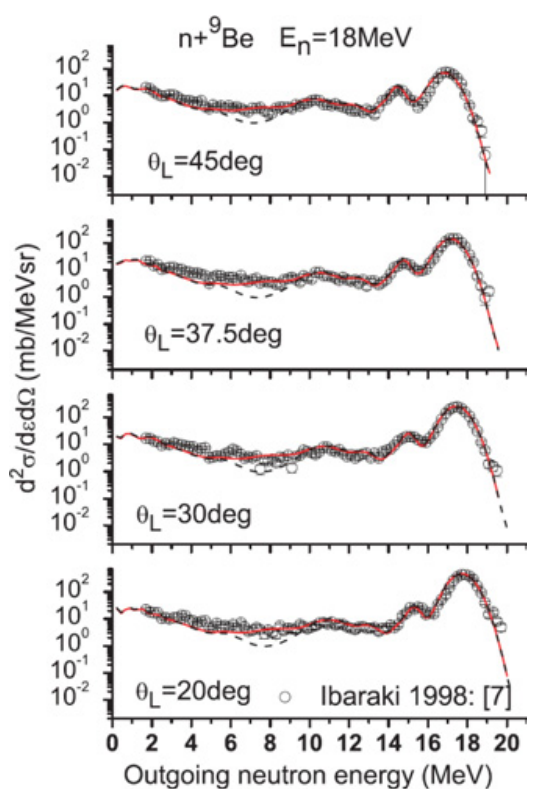

Figure 3. The double-differential cross sections for ${ }^{9} \mathrm{Be}(\mathrm{n}$, xn) at $E_{n}=18 \mathrm{MeV}$ for outgoing angles $20^{\circ}, 30^{\circ}, 37.5^{\circ}, 45^{\circ}$, respectively [5].

conservation is also kept strictly for the secondary particle emission process as Eq. (3). It is worth mentioning that the integral formula can largely reduce the size of the file- 6 in nuclear reaction databases.

\section{Results}

The model calculations of the DDCS of the total outgoing neutron had been performed for reactions such as ${ }^{12} \mathrm{C}(\mathrm{n}, \mathrm{xn}),{ }^{9} \mathrm{Be}(\mathrm{n}, \mathrm{xn}),{ }^{16} \mathrm{O}(\mathrm{n}, \mathrm{xn})$ and ${ }^{9} \mathrm{Be}(\mathrm{p}, \mathrm{xn})$. The calculated results for the different outgoing angles at different incident energies (taking $E_{n}=18 \mathrm{MeV}$ example in this paper) agree fairly well with the measurements as shown in Figs. 1-7. In these figures, the scattering points denote the experimental data. Similarly, the calculated total DDCS of outgoing neutrons at other angles also agree well with the experimental data.

It is worth mentioning that the contributions of two predicted energy levels $9.0\left(5 / 2^{+}\right)$and $10.0\left(5 / 2^{+}\right)$of ${ }^{9} \mathrm{Be}$ are added to obtain the DDCS of the total outgoing neutron as shown the solid lines in Figs. 3 and 4 [5] and Figs. 5 and 6 [2] both for neutron and proton induced ${ }^{9} \mathrm{Be}$ reactions. In these figures, the dashed lines denote the 


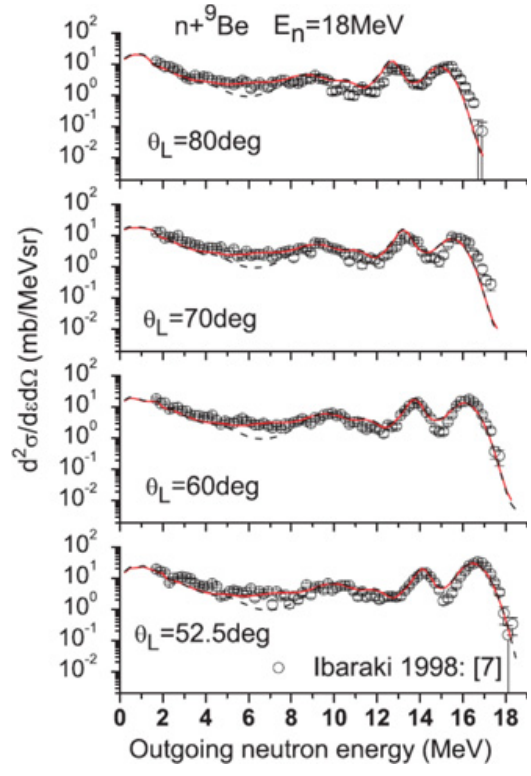

Figure 4. The same as Fig. 3, but for outgoing angles $52.5^{\circ}, 60^{\circ}$, $70^{\circ}, 80^{\circ}$, respectively [5].

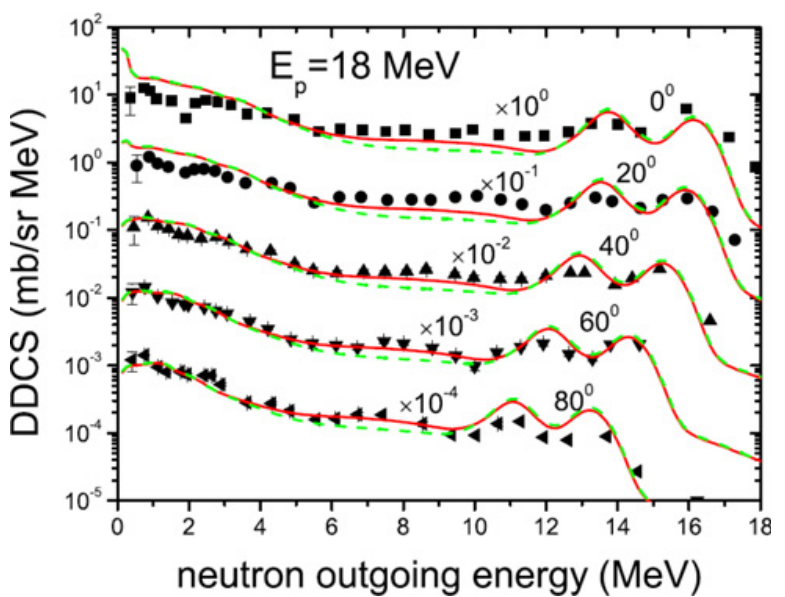

Figure 5. The double-differential cross sections for ${ }^{9} \mathrm{Be}(\mathrm{p}, \mathrm{xn})$ at $E_{p}=18 \mathrm{MeV}$ for outgoing angles $0^{\circ}, 20^{\circ}, 40^{\circ}, 60^{\circ}, 80^{\circ}$, respectively [2].

results only using the real energy levels. One can see that the calculated results of adding two predicted levels are in better agreement with the existing experimental data.

In addition, one can see that the calculated total DDCS of outgoing neutrons agree very well with the experimental data, except in low outgoing neutron energy regions at angles $0^{\circ}$ and $20^{\circ}$, as shown in Fig. 5. The reason is that the 4-mm-thick polyethylene beam stopper in the secondary Faraday cup slightly depresses the yields about $0.5-1.5 \mathrm{MeV}$ at forward angles [6].

For ${ }^{16} \mathrm{O}(\mathrm{n}, \mathrm{xn})$ reaction at low outgoing neutron energy regions as shown in Fig. 7, there are large discrepancies between the model calculations and the measurements. But the measurement is performed only by one group. To further validate our theory, we consider the effects of the charged particles using kerma coefficients in the frame of STLN. The kerma coefficient describes the kinetic energy of the charged particles released in matter, and expressed

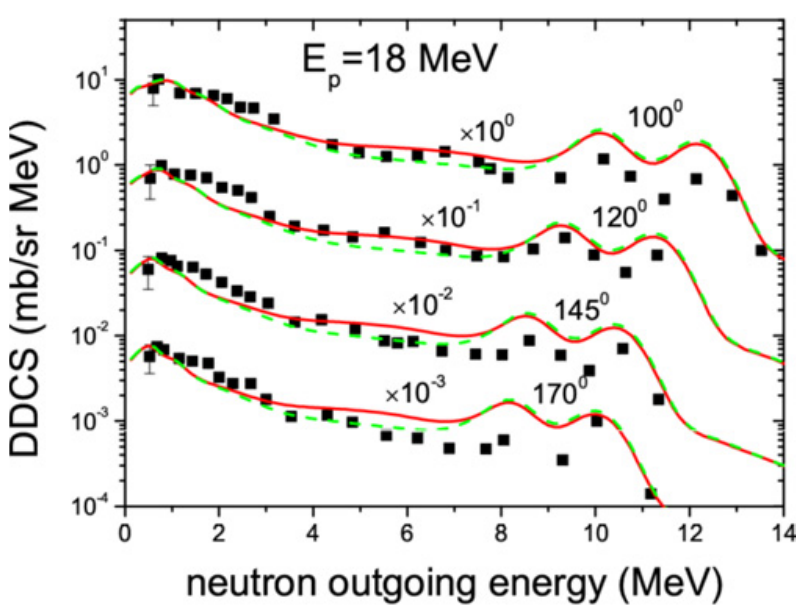

Figure 6. The same as Fig. 5, but for outgoing angles $100^{\circ}, 120^{\circ}$, $145^{\circ}, 170^{\circ}$, respectively [2].

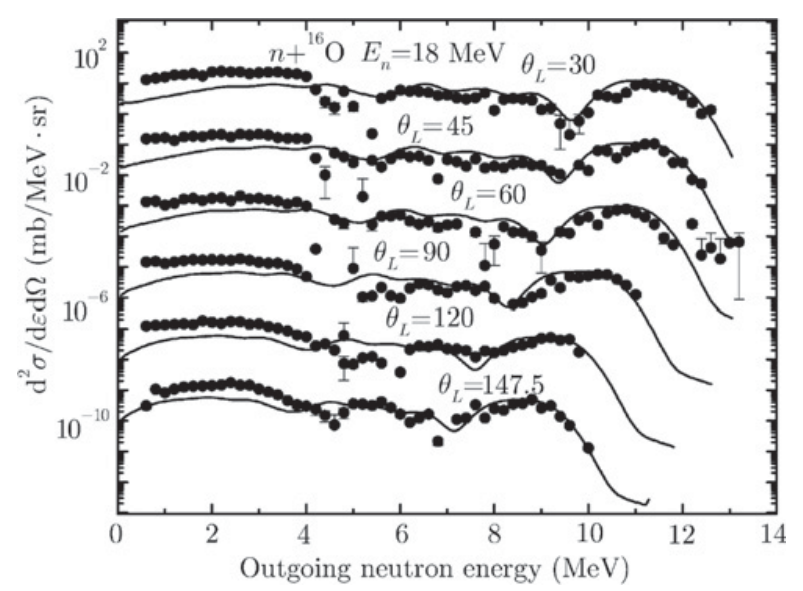

Figure 7. The double-differential cross sections for ${ }^{16} \mathrm{O}(\mathrm{n}$, xn $)$ at $E_{n}=18 \mathrm{MeV}$ for outgoing angles $30^{\circ}, 45^{\circ}, 60^{\circ}, 120^{\circ}, 147.5^{\circ}$, respectively [7].

as this equation

$$
k_{\Phi}\left(E_{n}\right)=\sum_{i} k_{\Phi}^{i}=N \sum_{i j k} \bar{E}_{i j k}\left(E_{n}\right) \sigma_{i j k}\left(E_{n}\right),
$$

where $\bar{E}_{i j k}$ and $\sigma_{i j k}$ are the average energy (expressed in $\mathrm{MeV}$ ) and the production cross section (in barns) in the lab system, respectively. Here, $i$ denotes the type of charged particle including light charged particles and recoiling nuclei, $j$ denotes the type of reaction channels, and $k$ indicates the excited energy level of the residual nucleus which can emit the secondary and/or third particle, or proceed via two-body separation, or carry out $\gamma$ decay. The coefficient $N$ converts the kerma coefficient from units of $\mathrm{MeV} \cdot \mathrm{b}$ to S.I. units of $\mathrm{fGy} \cdot \mathrm{m}^{2}$.

Figure 8 shows the calculated elastic recoiling kerma coefficients for neutron induced ${ }^{12} \mathrm{C}$ and ${ }^{16} \mathrm{O}$ reactions. And Figs. 9 and 10 show the total kerma coefficients of this work (solid line) for ${ }^{12} \mathrm{C}$ and ${ }^{16} \mathrm{O}$ vs incident neutron energies, respectively. The evaluated results of Ref. [9] are also shown as the dashed line. From Figs. 8-10, one can see that the calculated results agree well with the experimental data, especially at $18 \mathrm{MeV}$ incident energy. So it indirectly illustrates that our calculated results of the DDCS for ${ }^{16} \mathrm{O}(\mathrm{n}, \mathrm{xn})$ reaction at low outgoing neutron energy regions are reasonable, though there are large 


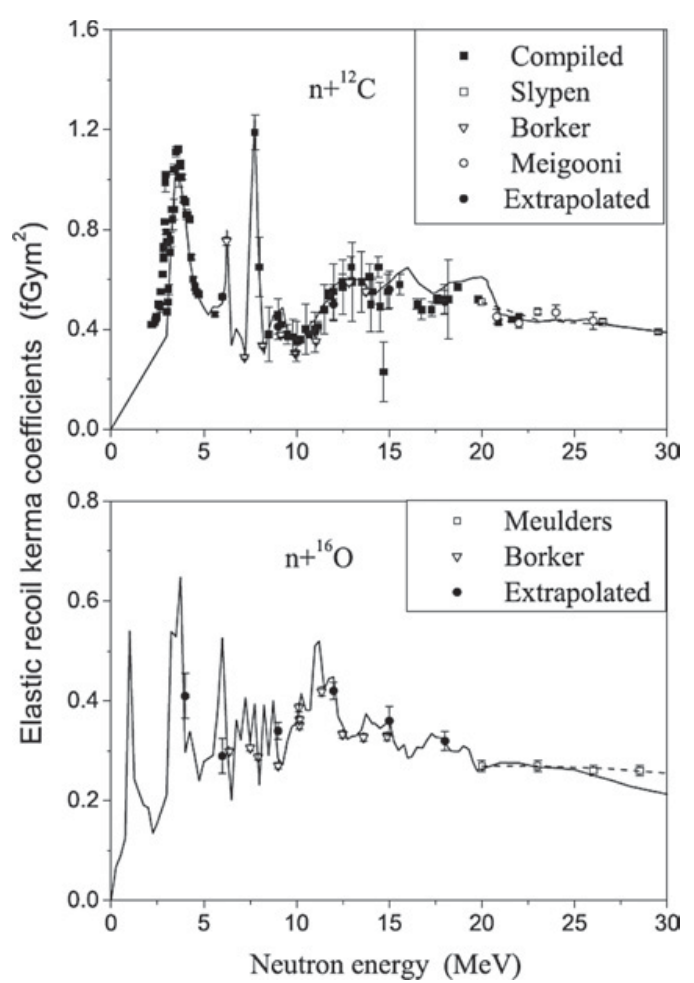

Figure 8. Elastic recoil kerma coefficients of this work (solid lines) for carbon and oxygen vs neutron incident energies [8].

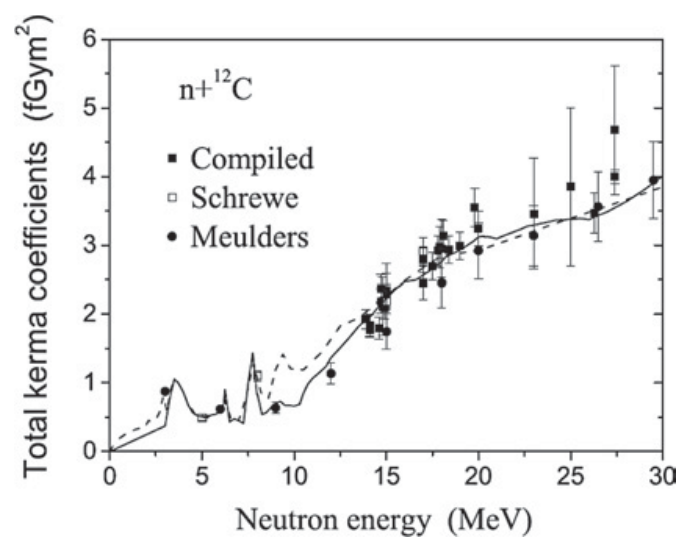

Figure 9. Total kerma coefficients of this work (solid line) for carbon vs incident neutron energies [8]. The evaluated results of Ref. [9] are shown as the dashed line.

discrepancies compared with the measurements at low outgoing neutron energy region.

\section{Summary}

STLN can successfully describe neutron induced nuclear reactions with $1 \mathrm{p}$-shell light nuclei, and had been used to

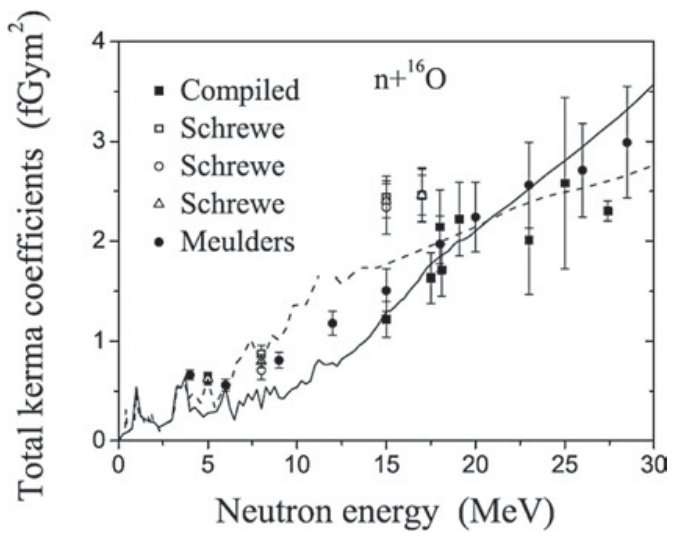

Figure 10. The same as Fig. 9, but for oxygen [8].

setup file-6 in CENDL. At same time, STLN is firstly used to describe ${ }^{9} \mathrm{Be}(\mathrm{p}, \mathrm{xn})$ reaction, and will be extended to describe $\mathrm{p}+{ }^{6,7} \mathrm{Li}$ reactions in the near future.

This work is supported by the National Natural Science Foundation of China (11465005); the Natural Science Foundation of Guangxi (2014GXNSFDA118003); Guangxi University Science and Technology Research Project (2013ZD007), and the project of outstanding young teachers' training in higher education institutions of Guangxi.

\section{References}

[1] A. Trkov, M. Herman, and D.A. Brown, ENDF-6 Formats Manual (Brookhaven National Laboratory, Upton, NY, 2011)

[2] Xiaojun Sun and Jingshang Zhang, Phys. Rev. C 93, 014609 (2016)

[3] Xiaojun Sun and Jingshang Zhang, Phys. Rev. C 92, 061601(R) (2015)

[4] Sun Xiao-Jun, Duan Jun-Feng, Wang Ji-Min, and Zhang Jing-Shang, Commun. Theor. Phys. 48, 534 (2007)

[5] Junfeng Duan, Jingshang Zhang, Haicheng Wu and Xiaojun Sun, Phys. Rev. C 80, 064612 (2009)

[6] V.V. Verbinski and W.R. Burrus, Phys. Rev. 177, 1671 (1969)

[7] Duan Jun-Feng, Yan Yu-Liang, Wang Ji-Min, Sun Xiao-Jun and Zhang Jing-Shang, Commun. Theor. Phys. 44, 701 (2005)

[8] Xiaojun Sun, Wenjing Qu, Junfeng Duan and Jingshang Zhang, Phys. Rev. C 78, 054610 (2008)

[9] M.B. Chadwick, H.H. Barschall, R.S. Caswell, et al., Med. Phys. 26, 974 (1999) 